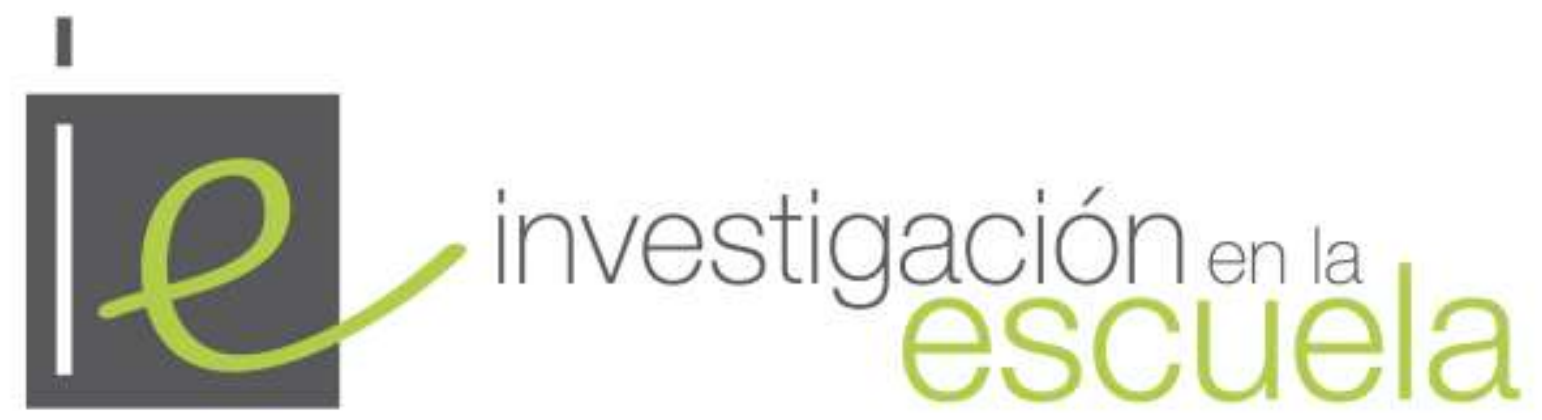

Revista internacional de investigación e innovación educativa

\title{
Programas de Formación Docente en Educación Superior en el contexto español ${ }^{1}$
}

\author{
Noelia Pérez Rodríguez? \\ Universidad de Sevilla \\ España
}

Citación: Pérez Rodríguez, N. (2019). Programas de Formación Docente en Educación Superior en el contexto español. Investigación en la Escuela, 97, 1-17.

Resumen: El presente estudio pretende realizar una revisión de los programas de formación docente llevados a cabo en las 50 universidades públicas españolas, mediante un análisis documental de fuentes. El objetivo principal es conocer las características de los diferentes programas formativos desarrollados, así como sus fortalezas y debilidades, con la finalidad de ofrecer una panorámica sobre la situación en esta área a nivel español. Los resultados extraídos, analizados a partir de un sistema de categorías "ad hoc", apuntan a la existencia de tres grandes tipos de programas: programas de noveles, cursos específicos y programas o títulos de formación en docencia universitaria. A su vez, cada uno de los programas está determinado por su duración, destinatarios y estrategias formativas. Las conclusiones más relevantes recogen la existencia mayoritaria de cursos específicos cortos sobre competencias o ejes docentes aislados sobre programas noveles o programas de formación en docencia universitaria, que se desarrollan en menor medida y no en todas las universidades. Este último resultado sugiere la necesidad de desarrollar, al menos, un

\footnotetext{
${ }^{1}$ Este trabajo es resultado parcial del Proyecto de I+D+i "La formación docente del profesorado universitario. Progresos y obstáculos de los participantes en un programa basado en ciclos de mejora de su práctica” (REF: EDU201675604-P) financiado por el Ministerio de Economía y Competitividad de España.

2 https://orcid.org/0000-0003-4375-4024
}

Página web: https://revistascientificas.us.es/index.php/IE Facebook: / Revista Investigación en la Escuela IE 
programa formativo de larga duración en cada universidad, que centre sus estrategias formativas en la reflexión y mejora de la práctica de aula y que permita mejorar la docencia tanto al profesorado novel como al profesorado experimentado.

Palabras clave: "Formación de docentes"; "Educación Superior"; "programa de formación"; "profesor de universidad".

\section{Teacher Training Programs in Higher Education in the Spanish context}

Abstract: This study aims to carry out a review of the teacher training programmes carried out in the 50 Spanish public universities, by means of a documentary analysis of sources. The main objective is to know the characteristics of the different training programs developed, as well as their strengths and weaknesses, in order to provide an overview of the situation in this area at the Spanish level. The results obtained, analyzed from a system of "ad hoc" categories, point to the existence of three main types of programmes: novice programmes, specific courses and university teaching programmes or qualifications. In turn, each of the programmes is determined by its duration, target groups and training strategies. The most relevant conclusions include the existence of specific short courses on competencies or isolated teaching axes on novice programs or training programs in university teaching, which are developed to a lesser extent and not in all universities. This last result suggests the need to develop at least one long-term training programme in each university, which focuses its training strategies on reflection and improvement of classroom practice and which allows both new and experienced teachers to improve teaching.

Key words: "Teaching training"; "Higher Education"; "training courses"; "university professors".

\section{Programmes de Formation des Enseignants de 1'Enseignement Supérieur dans le contexte espagnol}

Resumè: L'objectif de cette étude est de passer en revue les programmes de formation des enseignants mis en œuvre dans les 50 universités publiques espagnoles, au moyen d'une analyse documentaire des sources. L'objectif principal est de connaître les caractéristiques des différents programmes de formation développés, ainsi que leurs forces et faiblesses, afin de donner un aperçu de la situation dans ce domaine au niveau espagnol. Les résultats obtenus, analysés à partir d'un système de catégories "ad hoc", mettent en évidence l'existence de trois grands types de programmes: les programmes pour débutants, les cours spécifiques et les programmes ou qualifications d'enseignement universitaire. Chacun des programmes est à son tour déterminé par sa durée, ses groupes cibles et ses stratégies de formation. Parmi les conclusions les plus pertinentes, on peut citer l'existence de cours spécifiques de courte durée sur les compétences ou d'axes d'enseignement isolés sur les programmes pour débutants ou les programmes de formation en enseignement universitaire, qui sont développés dans une moindre mesure et pas dans toutes les universités. Ce dernier résultat suggère la nécessité de développer au moins un programme de formation à long terme dans chaque université, qui concentre ses stratégies de formation sur la réflexion et l'amélioration de la pratique en classe et qui permet aux nouveaux enseignants et aux enseignants expérimentés d'améliorer l'enseignement.

Mots-clés: "Formation des enseignants"; "Enseignement Supérieur"; "programme de formation"; "professeur d'université".

\section{Introducción}

En los últimos años los avances e investigaciones en Educación Superior ponen el foco en un modelo de enseñanza-aprendizaje centrado en el alumnado (Gargallo López, Fernández March y 
Jiménez Rodríguez, 2007; Marcelo, Yot, Mayor, Sánchez Moreno, Murillo, Rodríguez López y Pardo, 2014; Monroy y Hernández Pina, 2014). Dicho modelo supone tener en cuenta las ideas e intereses de los estudiantes en su propia formación y se vincula a su vez con un cambio de misión en la universidad, que enfrenta el reto de formar no solo a profesionales en su área de conocimiento, sino también a ciudadanos más comprometidos con su entorno (Martínez Martín, 2006). Tomando como referencia las investigaciones mencionadas, la implementación de dicho modelo supondría la consecución de una Educación Superior de calidad (European Comission, 2014). Considerar al alumnado un agente activo en la construcción del conocimiento requiere de un cambio sustancial en la propia enseñanza. Por tanto, la necesidad de mejorar la cualificación docente del profesorado universitario es cada vez más evidente.

La Ley Orgánica 6/2001, de 21 de diciembre, de Universidades (LOU) (2001) consideró relevante la formación de los docentes a nivel universitario, como una de las funciones que se integran dentro de su profesión. Su artículo 33.3 detalla: "La actividad y la dedicación docente, así como la formación del personal docente de las Universidades, serán criterios relevantes, atendida su oportuna evaluación, para determinar su eficiencia en el desarrollo de su actividad profesional" (p. 26).

Así mismo se recoge en el artículo 10 de la Conferencia Mundial sobre "La educación superior en el siglo XXI" (UNESCO, 1998) referida al "personal y los estudiantes, principales protagonistas de la educación superior":

Un elemento esencial para las instituciones de enseñanza superior es una enérgica política de formación del personal. Se deberían establecer directrices claras sobre los docentes de la educación superior, que deberían ocuparse, sobre todo, hoy en día, de enseñar a sus alumnos a aprender y a tomar iniciativas, y no a ser, únicamente, pozos de ciencia. (p. 26)

Por tanto, como venimos diciendo, los docentes deben formarse didácticamente para ajustarse al modelo de enseñanza actual, permitiendo que sea el propio alumnado el que construya el conocimiento.

A su vez, distintos grupos de investigación y organismos a nivel internacional profundizan en la relevancia de la formación docente a nivel universitario. Entre otros pueden destacarse el Centre for Teaching Excellence de la Universidad de Nueva York, el Centre of Expertise in Learning and Teaching (CELT) de la Universidad de Twente, el Centre for Research and Development of Higher Education de la Universidad de Helsinki o la European University Association (EUA).

En el contexto español también existen distintos grupos e investigaciones que profundizan en la formación docente del profesorado, con el objetivo de conseguir resultados que guíen las actuaciones formativas y que mejoren realmente desde la práctica la enseñanza y el aprendizaje en la universidad. Muestra de ello es el Grupo Interuniversitario en Formación Docente ${ }^{3}$ (GIFD) (Pagés, Hernández, Abadía, Bueno, Ubieto-Artur, Márquez, Sabaté y Jorba, 2016), formado por distintos investigadores de las universidades públicas de Cataluña o el Grupo de Formación e Innovación en Docencia Universitaria ${ }^{4}$ (FIDU) de la Universidad de Sevilla, en el que se enmarca este trabajo. Asimismo, también se han desarrollado asociaciones y redes como pueden ser la Asociación

\footnotetext{
${ }^{3}$ El grupo está integrado por investigadores de las Universidades de Barcelona (UB), Autónoma de Barcelona (UAB), Politécnica de Cataluña (UPC), Pompeu i Fabra (UPF), Girona (UdG), Lleida (UdL), Rovira i Virgili (URV) y Oberta de Catalunya (UOC).

${ }^{4}$ Grupo de Formación e Innovación en Docencia Universitaria (FIDU). Universidad de Sevilla (US)

https://grupofidu.com
} 
Iberoamericana de Docencia Universitaria ${ }^{5}$ (AIDU) o la Red Estatal de Docencia Universitaria ${ }^{6}$ (REDU).

Como puede verse, el contexto legal y científico presentado apoya la necesidad de profundizar en la formación didáctica del profesorado universitario. Para ello, parece oportuno en este momento analizar los actuales programas formativos llevados a cabo en las diversas universidades españolas, lo que nos dará una visión de conjunto del punto en el que nos encontramos y nos permitirá valorar sus fortalezas y debilidades. Desde ahí, definiremos los retos más importantes a abordar en la formación docente universitaria de nuestro país, de manera que avancemos de manera fundamentada y no intuitiva o descontextualizada.

\section{La formación pedagógica en la Educación Superior}

El profesorado universitario mayoritariamente se forma en un área de conocimiento específica, principalmente vinculada a la elaboración de su tesis doctoral y su posterior trayectoria investigadora, y muy excepcionalmente comienzan a dedicarse a la docencia en las aulas universitarias con formación pedagógica. Se enfrentan por tanto al ejercicio de una profesión tan compleja como la docencia, sin el manejo de herramientas, recursos y estrategias que faciliten su labor en el aula. El doble perfil profesional (investigador y docente) los distingue del resto de profesionales del ámbito educativo. El diseño de la carrera profesional en el ámbito universitario hace que en la mayoría de los casos se enfatice el primero de dichos perfiles, sin otorgar demasiada importancia al segundo y sin tener en cuenta, que dentro de esta carrera se encuentra el propio desarrollo profesional, el desarrollo educativo/pedagógico y el desarrollo personal (Sobrino García, 2015; Zabalza, 2006).

La falta de este desarrollo pedagógico hace que la mayoría de las prácticas docentes en la universidad se basen en la propia experiencia que los profesores han tenido durante su trayectoria académica como estudiantes (Clara Ventura, 2016; Triadó Ivern, Estebanell Minguell, Márquez Cebrián, Del Corral y De Villena, 2014), las cuales en su mayoría responden a un modelo transmisivo de la enseñanza que se aleja del modelo centrado en el estudiante que anteriormente hemos defendido.

Pero ¿por qué ocurre esto? Si analizamos las leyes que regulan la enseñanza superior podemos afirmar que no existe una regulación común de dicha formación. En la Ley Orgánica 11/1983 de Reforma Universitaria (LORU) no había ninguna mención a la formación del docente universitario. Sin embargo, en la Ley Orgánica 6/2001 de Universidades (LOU), vigente en la actualidad si podemos encontrar referencias a ella, como se han citado en páginas precedentes. No obstante, aunque en este caso sí se recoge, la idea es bastante general y tampoco se desarrollan acciones específicas, ni se detalla el tipo de formación, estrategias formativas u horas que debe acreditar el profesorado en este ámbito. De igual modo, no se describe cómo se evalúa dicha formación. La no especificación de esta formación hace que la realidad en cuanto a la formación docente sea diversa y compleja.

En la misma línea, el profesorado universitario declara la necesidad de formarse para mejorar su tarea docente, al igual que se encuentran motivados hacia la participación (en su mayoría voluntaria) en programas formativos (De la Cruz Tomé, 2000).

Tomando lo comentado como base distintos autores han profundizado en las competencias con las que debe contar el profesorado universitario, lo cual sería interesante considerar en el desarrollo de los programas formativos. Smith \& Simpson (1995) definieron 6 competencias básicas

\footnotetext{
${ }^{5}$ Asociación Iberoamericana de Docencia Universitaria (AIDU) http://www.asoc-aidu.com

${ }^{6}$ Red Estatal de Docencia Universitaria (REDU) https://red-u.org
} 
del profesorado: competencias educativas; competencias de planificación; competencias de gestión; competencias de comunicación y presentación; competencias de evaluación y relación y competencias interpersonales. Desde la visión del propio profesorado, Triadó Ivern et al. (2014) en una investigación en la que encuestaron a 2393 profesores/as de las 8 universidades públicas de Cataluña identificaron 8 competencias que los docentes señalaron dentro de su perfil profesional, siendo las dos más relevantes la competencia interpersonal y la competencia metodológica. Por tanto, desde la propia visión del profesorado se destaca la relevancia de desarrollar ambas competencias, las cuales encajarían dentro de su desarrollo educativo/pedagógico. No obstante, ¿se consideran en el desarrollo de los programas formativos dichas competencias de forma globalizada o por el contrario se aíslan entre sí?

Más allá de las competencias, son bastantes las investigaciones que han profundizado en la eficacia del uso de determinadas estrategias para el buen desarrollo de los programas formativos. Entre otras se propone partir de las concepciones del propio profesorado sobre la enseñanza y el aprendizaje, además de ofrecer una formación sistematizada (De la Cruz Tomé, 2003); la formación de equipos docentes que planifiquen y reflexionen conjuntamente (Gómez, Escofet y Freixa, 2014) organizados por departamento o titulación para que las actividades formativas estén más enfocadas en su práctica real y la formación se base en la interacción entre su propia disciplina científica, los contenidos que se enseñan y la formación pedagógica (De la Cruz Tomé, 2000); la renovación de la identidad del profesorado (Monereo y Domínguez, 2014); la investigación de la propia práctica como núcleo generador de calidad docente (Rodríguez, 2003); la creación de grupos de aprendizaje cooperativo con grabación de clases (Fernández March, 2003) y los sistemas de portafolios y carpetas docentes (De Rijdt, Tiquet, Dochy y Devolder, 2006; Fernández March, 2003).

En la actualidad, tal y como señala De la Cruz Tomé (2000) aunque no contamos con un programa institucionalizado a nivel nacional, España cuenta con cierta tradición en el ámbito de la formación docente universitaria. Según De la Cruz Tomé (2000) y Palomero (2003) este ámbito ha estado determinado, en gran medida, por tres hitos históricos:

El primero de ellos sería el seminario sobre "Pedagogía de la enseñanza superior", celebrado en Peñíscola en 1971; en segundo lugar, el seminario sobre "Pedagogía de urgencia para una universidad en crisis", coordinado por J. Bousquet en los años 1972/1973; y en tercer lugar, el curso titulado "Un nuevo método de formación de los profesores universitarios", desarrollado por el Instituto de Ciencias de la Educación (ICE) de la Universidad Autónoma de Madrid (UAM) en 1975 con la participación de M. Melnik de la Universidad de Massachussets. Tras estos tres hitos durante los años 80 comenzaron a desarrollarse multitud de encuentros, seminarios, jornadas y congresos que giraban en torno a la mejora de la formación docente del profesorado universitario en las distintas universidades españolas, todo ello influido por la LORU (1983).

Considerando la trayectoria de este campo, junto a los investigaciones comentadas, a continuación, profundizaremos en el estudio realizado, cuyos resultados presentan los distintos tipos de programas formativos que se llevan a cabo en las universidades españolas.

\section{Método}

El objetivo principal de este trabajo es conocer, por un lado, la existencia y características de los programas formativos desarrollados en las diferentes universidades españolas públicas, y por otro, detectar fortalezas y debilidades de las distintas modalidades formativas. El fin último es ofrecer una panorámica de los retos a abordar en esta área que favorezcan el desarrollo de medidas basadas en el análisis realizado. 
Para ello, se ha realizado una investigación de tipo cualitativa (Creswell, 2014) recurriendo a un análisis documental de fuentes, por ser la técnica que nos permite extraer la información de los distintos documentos existentes, realizando un análisis y síntesis de los mismos y estableciendo criterios de clasificación que permiten en última instancia encontrar elementos comunes y diferentes entre los programas mencionados.

\section{Muestra}

El estudio se centra en las 50 universidades públicas españolas (véase tabla 1). Se ha accedido a sus programas formativos a través de sus correspondientes Secretariados e Institutos de Formación del Profesorado. La muestra la componen 46 de ellas, pues los documentos relativos a los programas de la Universidad de las Islas Baleares, la Universidad Internacional Menéndez Pelayo, la Universidad Rey Juan Carlos y Universidad de Jaén, no estaban disponibles durante el momento de su consulta.

Tabla 1

Universidades que componen la muestra

Universidad Autónoma de Barcelona

Universidad Autónoma de Madrid

Universidad Carlos III de Madrid

Universidad Complutense de Madrid

Universidad de A Coruña

Universidad de Alcalá

Universidad de Alicante

Universidad de Almería

Universidad de Barcelona

Universidad de Burgos

Universidad de Cádiz

Universidad de Cantabria

Universidad de Castilla-La Mancha

Universidad de Córdoba

Universidad de Extremadura

Universidad de Girona

Universidad de Granada

Universidad de Huelva

Universidad de La Laguna

Universidad de La Rioja

Universidad de Las Palmas de Gran Canaria

Universidad de Murcia

Universidad de Oviedo

Universidad de Salamanca

Universidad de Santiago de Compostela

Universidad de Sevilla

Universidad de Valladolid

Universidad de Vigo

Universidad de Zaragoza

Universidad del País Vasco

Universidad Internacional de Andalucía

Universidad Miguel Hernández de Elche

Universidad Nacional de Educación a Distancia

Universidad Pablo de Olavide

Universidad Politécnica de Cartagena

Universidad Politécnica de Cataluña

Universidad Politécnica de Madrid

Universidad Politécnica de Valencia

Universidad Pompeu Fabra

Universidad Pública de Navarra

Universidad Rovira i Virgili

Universidad de Valencia

Universidad de León

Universidad Jaume I de Castellón

Universidad de Lleida

Universidad de Málaga

\section{Instrumento y procedimiento de análisis}

La consulta de los programas formativos nos ha permitido crear una clasificación basada en un sistema de categorías "ad hoc" en función de la información obtenida (San Martín, 2014). La recogida de datos se ha llevado a cabo durante los meses de septiembre-noviembre de 2018 y se ha realizado, como se ha mencionado, mediante el análisis de los documentos existentes en las páginas 
webs de los Secretariados de Formación e Institutos de Ciencias de la Educación de las universidades objeto de estudio.

La información analizada ha permitido establecer diferentes categorías y subcategorías. Para ello, conforme se consultaban las páginas webs de las diferentes universidades se realizó una tabla de clasificación que relacionaba los programas formativos y sus características. La información sintetizada fue exportada a Atlas.ti. Una primera visión de los datos permitía observar elementos comunes a los distintos programas que finalmente constituyeron las grandes categorías de análisis: tipo de titulación, destinatarios, duración y estrategias formativas. Focalizar en estas características facilitó diferenciar elementos dentro de las propias categorías. Ello permitió ir constituyendo las subcategorías. La relación entre categorías y subcategorías puede consultarse en la Tabla 2.

Tabla 2

Sistema de categorías

\begin{tabular}{ll}
\hline Categorías & Subcategorías \\
\hline Tipo de titulación & - Programas de formación para noveles \\
& - Títulos o programas de formación en docencia universitaria \\
& - Cursos específicos \\
\cline { 2 - 3 } Destinatarios & - Profesorado con menos de 5 años de experiencia docente \\
& - Profesorado con menos de 5 años de experiencia docente junto a \\
& becarios/contratados predoctorales \\
& - Profesorado con menos de 5 años de experiencia docente junto a \\
& becarios/contratados predoctorales/profesores asociados o \\
& ayudantes \\
& - Todo el Personal Docente e Investigador \\
& - Cortos (75 horas o menos) \\
Duración & - Largos \\
Estrategia formativa & - Mentoría \\
& - Análisis y reflexión de su propia práctica de aula \\
& - Observaciones de aula \\
& - Carpetas docentes/portafolios \\
& - Cursos generales
\end{tabular}

Para el análisis de los datos nos hemos apoyado en el programa de análisis cualitativo Atlas.ti v.8.4.0.

\section{Resultados}

Los resultados se presentan siguiendo el sistema de categorías anterior, en función a la categoría y subcategorías mencionadas. Es importante destacar que las categorías no son excluyentes.

\section{Tipo de titulación}

Si atendemos al tipo de formación existente encontramos a su vez programas de formación para docentes noveles, títulos o programas de formación en docencia universitaria y cursos específicos. 
Todas las universidades objeto de análisis ofertan cursos específicos a lo largo del año. En un porcentaje menor, se ofertan programas para noveles (39,1\% de las universidades) y títulos o programas formativos en docencia universitaria $(10,9 \%)$.

Las 18 universidades que ofrecen programas formativos dirigidos directamente a profesorado universitario novel se recogen en la Tabla 3.

Tabla 3

Universidades que ofrecen programas para docentes noveles

\begin{tabular}{cl}
\hline Universidades & \\
\hline 1. Universidad Autónoma de & 10. Universidad de Salamanca \\
2. Univelona & 11. Universidad de Valladolid \\
3. Universidad de A Coruña & 12. Universidad de Zaragoza \\
4. Universidad de Almería & 13. Universidad de Miguel Hernández de \\
5. Universidad de Burgos & Elche \\
6. Universidad de Córdoba & 14. Universidad Politécnica de Madrid \\
7. Universidad de Extremadura & 15. Universidad Pública de Navarra \\
8. Universidad de Granada & 16. Universidad Jaume I de Castellón \\
9. Universidad de Málaga & 17. Universidad de Murcia \\
\hline
\end{tabular}

Los cursos específicos de formación que a grosso modo se agrupan en competencias o en ejes temáticos, considerando por separado docencia, gestión e investigación. Por exponer algún ejemplo, en la Universidad Autónoma de Barcelona, los cursos ofertados se organizan en función de las competencias que desarrolla: interpersonal, metodológica, comunicativa, de planificación y gestión de la docencia, de trabajo en equipo/ innovación. En la Universidad Carlos III de Madrid los cursos se organizan en ejes (didáctico, investigador y transversal). Dentro de la competencia docente son comunes los cursos centrados en metodologías, así como en el uso de las tecnologías en el aula.

Además de los cursos específicos, algunas universidades llevan a cabo títulos o programas de formación en docencia universitaria con diferentes denominaciones, como ya decíamos. De las 46 universidades solo $5(10,9 \%)$ recogen este tipo de formación. En la Tabla 4 se recogen las universidades y la denominación de los títulos respectivos.

Tabla 4

Relación de universidades y denominación de los titulos especificos de formación en docencia universitaria

\begin{tabular}{ll}
\hline Universidad & Denominación \\
\hline Universidad Autónoma de Madrid & Título de experto en docencia universitaria \\
Universidad Politécnica de Valencia & Título de Experto en Pedagogía Universitaria (EUPU) \\
Universidad de Rovira i Virgili & Curso de especialista en Docencia Universitaria \\
Universidad de Lleida & Especialista en Docencia Universitaria \\
Universidad de Sevilla & Programa de Formación e Innovación Docente del \\
& Profesorado (FIDOP) \\
\hline
\end{tabular}

\section{Destinatarios}

Si además de considerar los tipos de titulación existentes, observamos quiénes son los destinatarios, encontramos que todas las universidades dirigen su formación a todo el Personal Docente e Investigador (PDI), en el caso de los cursos específicos y a los títulos o programas formativos en docencia universitaria. 
Sin embargo, realizan una especificación en relación a los destinatarios de los programas noveles. De los 16 programas noveles ${ }^{7}$ de los que contamos con información sobre los destinatarios, el 31,3\% está dirigido a profesorado con menos de 5 años de experiencia; el 31,3\% a profesorado con menos de 5 años de experiencia más becarios o contratados predoctorales; el 12,5\% se dirige a profesorado con menos de 5 años de experiencia, becarios o contratados predoctorales y profesores/as asociados o ayudantes doctores. El 25\% de estos programas se dirige a todo el Personal Docente e Investigador (véase Figura 1). Para conocer la relación de programas noveles y destinatarios por universidades puede consultarse la Figura 2.

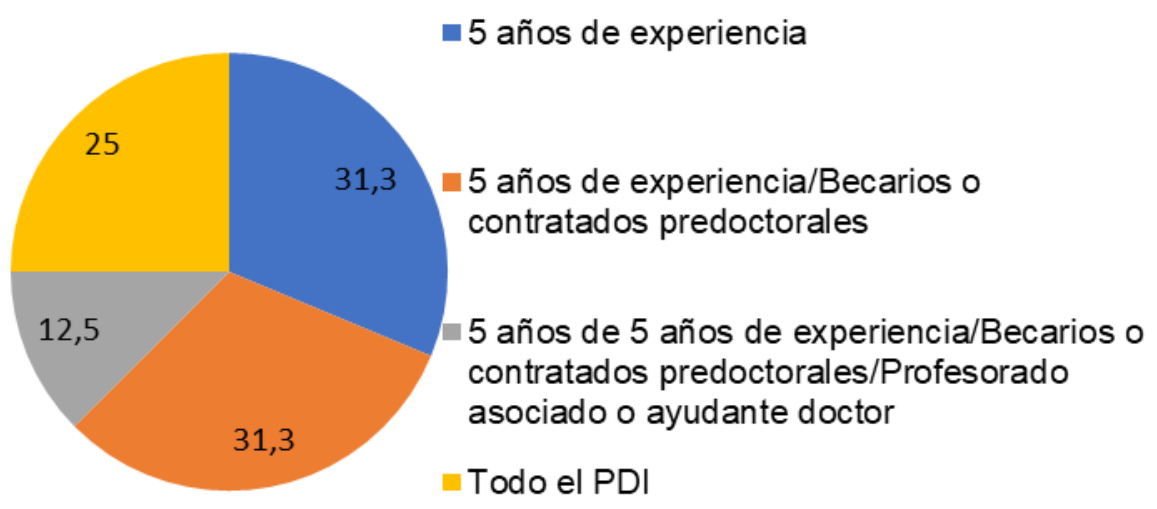

Figura 1. Porcentajes relación programas de formación noveles y destinatarios

${ }^{7}$ No existe información sobre los destinatarios de las Universidades de Valladolid y Miguel Hernández de Elche. 


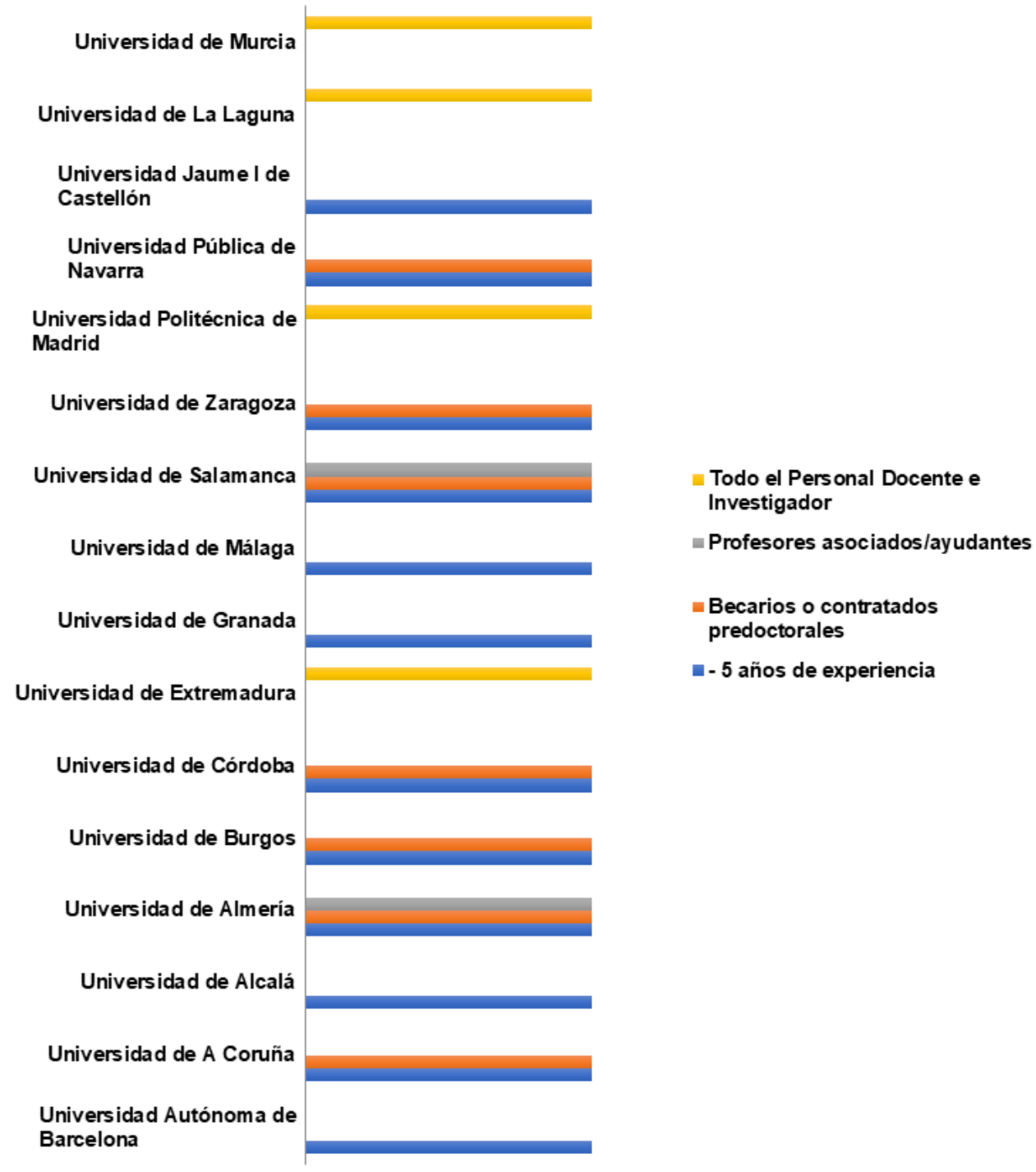

Figura 2. Programas de formación inicial y destinatarios por universidades.

Nota: La Universidad de Murcia da preferencia a profesores asociados y a aquellos que tengan menos de 2 años de experiencia docente.

\section{Duración}

Otra de las categorías emergentes que se ha considerado ha sido la duración de los diferentes programas formativos. Se ha asignado una formación corta a aquella que tiene 75 horas o menos y una duración larga a aquella de más de 75 horas.

Los datos muestran como los cursos específicos existentes en todas las universidades son de corta duración. Del resto de programas contamos con información de 19 de ellos ${ }^{8}, 14$ programas de formación para noveles y $4^{9}$ títulos o programas de formación en docencia universitaria. Del total, 12

${ }^{8}$ No contamos con la información sobre la duración de los programas de formación para noveles de la Universidad Pública de Navarra, Alcalá y Valladolid.

La Universidad Jaume I de Castellón especifica una duración de 2 cursos, aunque no declara horas por lo que no se ha tenido en cuenta en relación a esta categoría.

${ }_{9}^{9}$ No contamos con información del programa desarrollado por la Universidad de Lleida. 
programas cuentan con una duración larga. Los 7 restantes son de duración corta y se corresponden con programas de formación para noveles. Algunos programas para noveles como el de la Universidad de Córdoba y la Politécnica de Madrid miden su formación en ECTS, aunque también se corresponden con una duración larga. El programa novel desarrollado en la Universidad de Córdoba, además de la duración en ECTS, especifica que se desarrolla durante 2 cursos académicos, algo que no se declara en el resto. En la Tabla 5 se presenta una relación de los programas para noveles por universidades en función de su duración.

Tabla 5

Programas dirigidos a etapa inicial en función de la duración

\begin{tabular}{ll}
\hline Cortos & Largos \\
\hline Universidad de Almería & Universidad Autónoma de Barcelona \\
Universidad de Burgos & Universidad de A Coruña \\
Universidad de Extremadura & Universidad de Granada \\
Universidad de Salamanca & Universidad de Málaga \\
Universidad de Zaragoza & Universidad de Miguel Hernández de Elche \\
Universidad de Murcia & Universidad de Córdoba \\
& Universidad Politécnica de Madrid \\
\hline
\end{tabular}

Nota 1: La Universidad de Córdoba mide su formación en 18 ECTS (2 cursos).

Nota 2: La Universidad Politécnica de Madrid mide su formación en 10 ECTS en el caso de completar las fases de módulos o en 15 ECTS si además realizan la fase de practicum.

De los 4 títulos o programas de formación en docencia universitaria de los que contamos con información, 3 de ellos (Universidad Autónoma de Madrid, Politécnica de Valencia y Rovira Virgili) también miden su formación en ECTS. Además, al ser una formación de larga duración consideran cursos académicos para su realización, como ocurría con el programa para noveles de la Universidad de Córdoba. El programa FIDOP de la Universidad de Sevilla si mide la formación en horas desarrolladas a lo largo de un curso académico. En la Tabla 6 se presentan los programas descritos por universidades en relación a su duración en años o cursos académicos, así como en horas o ECTS.

Tabla 6

Relación entre programas, duración (años o curso académicos) y ECTS/ horas

\begin{tabular}{|c|c|c|}
\hline Universidad & $\begin{array}{l}\text { Duración } \\
\text { (años/cursos } \\
\text { académicos) }\end{array}$ & ECTS/horas \\
\hline $\begin{array}{l}\text { Universidad } \\
\text { Autónoma de } \\
\text { Madrid }\end{array}$ & $\begin{array}{l}2 \text { cursos } \\
\text { académicos } \\
\text { para la } 1^{\mathrm{a}} \text { fase y } \\
1 \text { curso } \\
\text { académico para } \\
\text { la } 2^{\mathrm{a}} \text { fase. }\end{array}$ & $\begin{array}{l}\text { Está compuesto de "dos fases": } \\
\text { 1 }^{a} \text { fase: } 10 \text { créditos ECTS de cursos de formación continua (6 } \\
\text { ámbito metodológico y } 4 \text { del resto). } \\
2^{a} \text { fase: } 15 \text { ECTS. Proyecto de "cambio docente". }\end{array}$ \\
\hline $\begin{array}{l}\text { Universidad } \\
\text { Politécnica de } \\
\text { Valencia }\end{array}$ & $\begin{array}{l}\text { Un año a } \\
\text { tiempo } \\
\text { completo } \\
\text { (septiembre- } \\
\text { junio) }\end{array}$ & $\begin{array}{l}20 \text { ECTS distribuidos en: } \\
\text { - Formación obligatoria y optativa (15 ECTS) } \\
\text { - Practicum ( } 3 \text { ECTS) } \\
\text { - Trabajo final (2 ECTS) }\end{array}$ \\
\hline
\end{tabular}




\begin{tabular}{lll}
\hline Universidad & $\begin{array}{l}\text { Duración } \\
\text { (años/cursos } \\
\text { académicos) }\end{array}$ & ECTS/horas \\
\hline Universidad de & Un año y & 30 ECTS organizados en 9 módulos. \\
Rovira i Virgili & medio & 100 horas: \\
Universidad de & Un curso & -90 horas: curso General de Docencia Universitaria (GDU) \\
Sevilla & académico & -40 presenciales y 50 no presenciales. \\
& & -10 horas - presenciales y obligatorias - de participación en \\
& las Jornadas FIDOP.
\end{tabular}

\section{Estrategia formativa}

Además de compartir la duración o los destinatarios, los programas y cursos siguen distintas estrategias formativas. Las estrategias comunes a los distintos programas encontradas se pueden consultar en la Tabla 7.

Tabla 7

Estrategias formativas llevadas a cabo

-Cursos generales sobre ejes o competencia

-Procesos de mentorización o guía

-Análisis y reflexión sobre la práctica

-Observaciones de aula

-Elaboración de carpetas docentes/portafolios

Los cursos específicos de las 46 universidades comparten estrategia y se centran en desarrollar un eje o competencia docente no conectada con las demás. Del resto de los 23 programas (noveles y de docencia universitaria) solo contamos con información sobre la estrategia formativa seguida en 19 de ellos (82,6\%). No obstante, a excepción de los programas para noveles de las Universidades de A Coruña, Zaragoza y La Laguna y el programa de docencia universitaria de la Universidad de Rovira i Virgili que solo se centran en cursos generales sobre ejes o competencias, el resto combina diferentes estrategias formativas, siendo compartidas las estrategias en algunas ocasiones tanto por los programas de formación para noveles como por los títulos o programas de formación en docencia universitaria, tal y como se presentará a continuación.

De todas las combinaciones las más frecuentes son realizar análisis y reflexión de su propia práctica junto a cursos generales centrados en ejes o competencias docentes. Es la estrategia seguida por 4 programas, 3 programas noveles (Universidad de Málaga, Murcia y Pública de Navarra) y un programa de formación en docencia universitaria (Universidad de Lleida).

Seguido de ello, otros programas combinan estrategias como la mentoría o tutorización, el análisis y reflexión sobre la práctica, la realización de carpetas docentes/portafolios con cursos generales sobre ejes o competencias como ocurre en los programas para noveles de la Universidad de Almería y Burgos, además de en el programa de formación en docencia universitaria de la Universidad Politécnica de Valencia. Otras universidades siguen las mismas estrategias comentadas anteriormente pero sin incluir carpetas docentes/portafolios. Concretamente ocurre en los programas noveles de la Universidad de Córdoba, Politécnica de Madrid y Jaume I de Castellón.

Por su parte, el programa novel desarrollado por la Universidad de Salamanca o el programa de formación docente de la Universidad de Sevilla combinan la mentorización, con el análisis y reflexión sobre su propia práctica y el desarrollo de carpetas docentes o portafolios. Aunque estas 
dos universidades comparten estrategias formativas, el programa de la Universidad de Sevilla, además de usar las estrategias comentadas, también incluye el uso de una estrategia que no comparte con la anterior universidad, denominada Ciclos de Mejora Docente (CMD) (Porlán, 2017), en el que los docentes participantes en el programa diseñan innovaciones sobre sus asignaturas durante las clases presenciales del curso y las implementan en el aula.

El programa para noveles de la Universidad Autónoma de Barcelona además de combinar cursos sobre competencias con el desarrollo de carpetas docentes/portafolios, también incluye observaciones de aula por pares entre los propios compañeros/as participantes en el programa.

Por su parte, el programa de formación en docencia universitaria de la Universidad Autónoma de Madrid combina estrategias como el análisis y reflexión de su propia práctica, con la elaboración de carpetas docentes/portafolios y la inclusión de cursos específicos sobre ejes o competencias.

El programa de noveles desarrollado por la Universidad de Extremadura se desarrolla a través de cursos centrados en estrategias y competencias junto a procesos de mentorización.

De entre las características comentadas, cabe mencionar que en el caso de las estrategias de mentorización en algunas ocasiones el profesorado mentor o tutor debe tener vinculación contractual permanente con la universidad y contar con una evaluación Docentia positiva, como ocurre en la Universidad de Burgos.

Si aislamos cada una de la estrategias, y exceptuando el trabajo en torno a ejes y competencias, lo más frecuente sería el análisis y reflexión sobre la propia práctica y lo menos la estrategia de observación de aula (ya sea propia o entre pares). Entre estos extremos se encontrarían, de mayor a menor, los procesos de mentorización o guía y las carpetas docentes y portafolios (véase Figura 3).

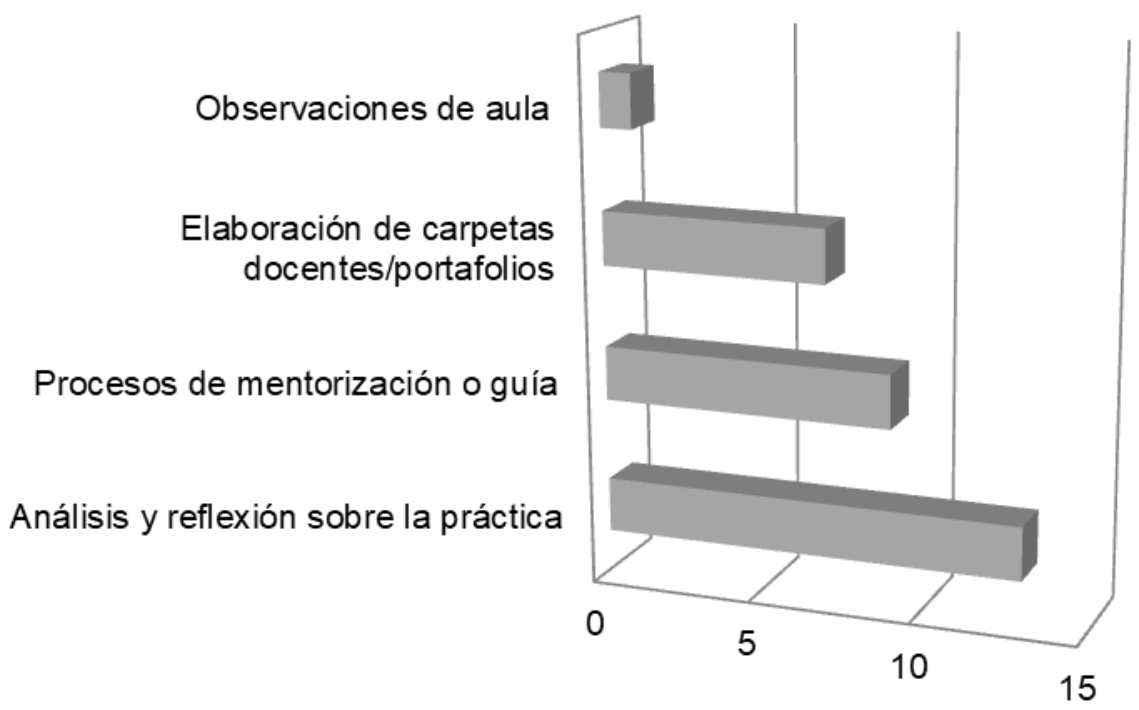

Figura 3. Estrategias formativas en grado de frecuencia

\section{Discusión y Conclusiones}

$\mathrm{Al}$ considerar los resultados en relación con las distintas categorías objeto de análisis, en cuanto a los tipos de titulación es llamativo que lo más frecuente es la realización de cursos específicos, así como que las universidades en las que existen programas de formación noveles no cuentan con programas de formación en docencia universitaria y viceversa. Esto se relaciona 
directamente con la segunda categoría que se centraba en analizar los destinatarios de las distintas formaciones. El 25\% de los programas noveles están dirigidos a todo el PDI mientras el resto solo se dirigen a personal con menos de 5 años de experiencia, becarios o contratados predoctorales y profesores asociados/ayudantes. Por tanto, ¿qué ocurre con el PDI de estas universidades que tienen más años de experiencia y quieren mejorar su práctica docente?

La tercera de las categorías muestra como todos los cursos específicos son de corta duración. Los datos también reflejan como todos los programas de formación en docencia universitaria son de duración larga, algo que no ocurre con la totalidad de programas de formación novel.

Con respecto a las estrategias, sigue siendo frecuente la estrategia centrada en competencias o ejes específicos, frente a la combinación de diferentes estrategias por algunos programas noveles y de formación en docencia universitaria. No obstante, si aislásemos las distintas estrategias lo más frecuente es el análisis y reflexión sobre la práctica, aunque dicha estrategia no siempre está unida a la elaboración de carpetas docentes o portafolios. Sin embargo, el estudio realizado por De Rijdt et al. (2006) muestra como los profesores universitarios que utilizan el portafolio reflexionan más sobre su enseñanza, replanteándose cambios sobre sus materiales o metodología. Por tanto, puede resultar de interés buscar una mayor vinculación entre ambas estrategias y usar los portafolios como una herramienta de análisis sobre la propia práctica, de forma que ambas estrategias estén vinculadas y se potencien mutuamente.

Si tuviésemos que establecer la pauta más frecuente de formación del profesorado universitario tras el análisis realizado se podría afirmar que lo más frecuente son los cursos específicos, de corta duración, dirigidos a todo el PDI y centrados en ejes o competencias docentes. A ello le seguirían los programas noveles, de corta duración, centrados en ejes y competencias y dirigido a profesorado con menos de 5 años de experiencia docente junto a becarios/contratados predoctorales. Si las investigaciones declaran la necesidad de cambiar de paradigma educativo (Cid, Pérez y Zabalza, 2013), acercando la universidad al modelo de enseñanza centrado en el alumnado, ¿por qué solo 23 de las 46 universidades analizadas tienen programas específicos dirigidos a mejorar la docencia universitaria?

Las conclusiones de esta investigación nos han permitido reforzar la idea de que las acciones formativas que se llevan a cabo en la actualidad no están contextualizadas en las prácticas reales y de aula del propio profesorado. Aunque algunos autores (Cabalín y Navarro, 2010; Triadó Ivern et. al, 2014) indican la necesidad de desarrollar en el profesorado una serie de competencias que partan de las que ya tienen (Álvarez-Rojo et al., 2009) para incidir y mejorar aquellas competencias en las que tienen carencias, los cursos de formación permanente no suelen partir de esta premisa ya que, como se ha presentado en los resultados, es frecuente la oferta de cursos de máximo 40/50 horas en los que reciben formación poco vinculada a su práctica y desconectada de sus experiencias de aula. Además, el desarrollo de las competencias se presenta en su mayoría de forma parcelada, en ejes temáticos o competencias docentes específicas, lo que realmente no favorece el desarrollo de las mismas de una forma integral.

Tomar de base las investigaciones que apuntan a las carencias psicopedagógicas del profesorado universitario debería hacer repensar sobre la necesidad de la existencia de, al menos, un programa específico de formación docente por universidad, dirigido a ser posible a todo el PDI. Ello permitiría, tanto al profesorado novel como al profesorado experimentado, poder mejorar su práctica de aula, alejándose de realizar únicamente cursos de formación permanente sobre competencias docentes aisladas. Sería, por tanto, necesario incorporar procesos de formación que, basándose en resultados de investigaciones, sean más estables y estructurados (De la Cruz Tomé, 2003; Gómez, Escofet y Freixa, 2014; Monereo y Domínguez, 2014; Rodríguez, 2003).

Finalmente, y apoyando la idea de De la Cruz Tomé (2000, 2003), la diversidad de tipologías y estrategias formativas llevadas a cabo en Educación Superior (muchas veces desconocidas entre sî) 
sugieren la necesidad de institucionalizar la formación docente universitaria y formar redes o equipos docentes que cuenten con un modelo formativo que realmente se base en los resultados de las investigaciones desarrolladas en este campo.

\section{Referencias}

Álvarez-Rojo, V., Asensio-Muñoz I., Clares, J., Del-Frago, R. García-Lupión, B., García-Nieto, N., García-García, M., Gil, J. González-González, D., Guardia, S., Ibarra, M., López-Fuentes, R., Rodríguez-Diéguez, A., Rodríguez-Gómez, G., Rodríguez-Santero, J., Romero, S. y Salmerón, P. (2009). Perfiles docentes para el espacio europeo de educación superior (EEES) en el ámbito universitario español. RELIEVE, 15 (1), 1-18.

Cabalín, D. y Navarro, N. (2010). Conceptualización de los estudiantes sobre el buen profesor universitario en las carreras de la salud de la Universidad de La Frontera - Chile. Int. J. Morphol, 26, 887-892.

Cid Sabucedo, A., Pérez Abellás, A. y Zabalza Beraza, M. A. (2013). Las prácticas de enseñanza realizadas/observadas de los «mejores profesores» de la Universidad de Vigo. Educación XX1, 16 (2), 265-296. Recuperado de https://doi.org/10.5944/educxx1.16.2.2643.

Clara Ventura, A. (2016). ¿Enseño como aprendí?: el rol del estilo de aprendizaje en la enseñanza del profesorado universitario. Aula abierta, 44, 91-98. DOI: http://dx.doi.org/10.1016/j.aula.2016.05.001.

Creswell, J.W. (2014). Research Design: Qualitative, Quantitative and Mixed Methods Approaches. Los Angeles: Sage.

De la Cruz Tomé, M. (2000). Formación pedagógica inicial y permanente del profesor universitario en España: reflexiones y propuestas. Revista Interuniversitaria de Formación del Profesorado, 38, 19-35.

De la Cruz Tomé, M. A. (2003). Necesidad y objetivos de la formación pedagógica del profesor universitario. Revista de Educación, (331), 35-66.

De Rijdt, C., Tiquet, E., Dochy, F. \& Devolder, M. (2006). Teaching portfolios in higher education and their effects: An explorative study. Teaching and Teacher Education, 22, 1084-1093. Recuperado de https://doi.org/10.1016/j.tate.2006.07.002.

European Comission. (2014). High Level Group on the Modernisation of Higher Education. European Comission. Luxembourg. Recuperado de http://ec.europa.eu/dgs/education_culture/repository/education/library/reports/modernisati on-universities_en.pdf

Fernández March, A. (2003). Formación pedagógica y desarrollo profesional de los profesores de universidad: análisis de las diferentes estrategias. Revista de Educación, (331), 171-197.

Gargallo López, B., Fernández March, A. y Jiménez Rodríguez, M. A. (2007). Modelos docentes de los profesores universitarios. Teoría de la educación, 19, 167-189.

Gómez, M., Escofet, A., y Freixa, M. (2014). Los equipos docentes en la educación superior ¿Utopía o realidad? Revista Española de Pedagogía, (259), 509-523.

Ley Orgánica 11/1983, de 25 de agosto, de Reforma Universitaria. Boletín Oficial del Estado, núm. 209, de 1 de septiembre de 1983, pp. 24034- 24042. Recuperada de https://www.boe.es/boe/dias/1983/09/01/pdfs/A24034-24042.pdf.

Ley Orgánica 6/2001, de 21 de diciembre, de Universidades. Boletín Oficial del Estado, núm. 307, de 24 de diciembre de 2001, pp. 49400-49425. Recuperada de https://www.boe.es/boe/dias/2001/12/24/pdfs/A49400-49425.pdf.

Marcelo, C., Yot, C., Mayor, C. Sánchez Moreno, M., Murillo, P., Rodríguez López, J. M. y Pardo, A. (2014). Las actividades de aprendizaje en la enseñanza universitaria: ‘hacia un aprendizaje 
autónomo de los alumnos? Revista de Educación, 363 (enero-abril), 334-359. DOI: 10.4438/1988592X-RE-2012-363-191.

Martínez Martín, M. (2006). Formación para la Ciudadanía y Educación Superior. Revista Iberoamericana de Educación, (42), 85-102.

Monereo, C. y Domínguez, C. (2014). La identidad docente de los profesores universitarios competentes. Educación XX1, 17 (2), 83-104. DOI: 10.5944/educxx1.17.2.11480.

Monroy, F. y Hernández Pina, F. (2014). Factores que influyen en los enfoques de aprendizaje universitario. Una revisión sistemática. Educación XX1, 17 (2), 105-124. DOI: 10.5944/educxx1.17.2.11481.

Pagés, T., Hernández, C., Abadía, A. R., Bueno, C. Ubieto-Artur, I., Márquez, D., Sabaté, S. y Jorba, H. (2016). La innovación como competencia docente en la universidad: Innovación orientada a la mejora de aprendizaje. Revista de Psicologia, Ciències de l'Educació i de l'Esport, 34 (1), 33-43.

Palomero, J. E. (2003). Breve historia de la formación psicopedagógica del profesorado universitario en España. Revista Interuniversitaria de Formación del Profesorado, 17 (2), 21-41.

Porlán, R. (Coord.) (2017). Enseñanza universitaria. Cómo mejorarla. Madrid: Morata.

Rodríguez, S. (2003). Nuevos retos y enfoques en la formación del profesorado universitario. Revista de Educación, (331), 67-69.

San Martín, D. (2014). Teoría fundamentada y Atlas.ti: recursos metodológicos para la investigación educativa. Revista electrónica de investigación educativa, 16(1), 104-122. Recuperado de http://www.scielo.org.mx/scielo.php?script=sci_arttext\&pid=S160740412014000100008\&lng=es\&tlng=es.

Smith, K. S. \& Simpson, R. D. (1995). Validating teaching competencies for Faculty Members in Higher Education: a national study using the Delphi Method. Innovative Higher Education, 3 (19), 223-234.

UNESCO. (1998). Conferencia Mundial sobre la Educación Superior. La educación superior en el siglo XXI. Visión y acción. París.

Zabalza, M. (2006). Competencias docentes del profesorado universitario: calidady desarrollo profesional. Madrid: Narcea.

\section{Información sobre los autores}

Autor: Noelia Pérez Rodríguez

Institución: Universidad de Sevilla

Email: nperez4@us.es 


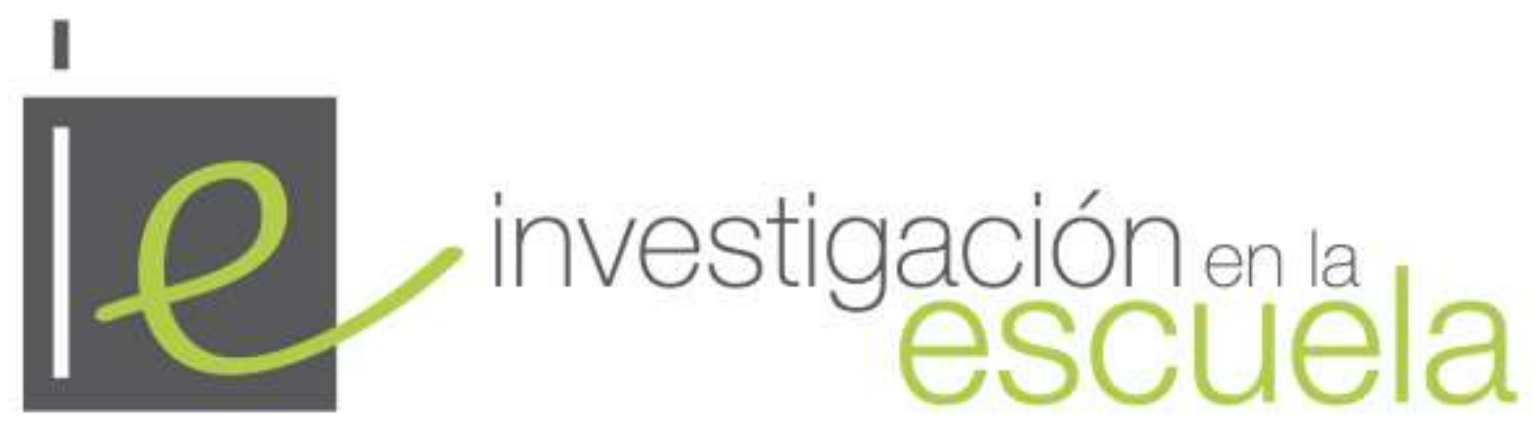

Revista académica evaluada por pares y de acceso abierto

Número 97

30 de abril de 2019

ISSN 2443-9991

\section{(c)}

SOMREFIIGHISRESERVEDLOS/as lectores/as pueden copiar, mostrar, y distribuir este artículo, siempre y cuando se de crédito y atribución al autor/es y a Investigación en la Escuela, se distribuya con propósitos no-comerciales, no se altere o transforme el trabajo original. Más detalles de la licencia de CreativeCommons se encuentran en http://creativecommons.org/licenses/by-nc-sa/3.0 Cualquier otro uso debe ser aprobado en conjunto por el autor/es, o Investigación en la Escuela.

Uิ investigacion-en-la-escuela

Contribuya con comentarios y sugerencias en la web de la revista. Por errores y sugerencias contacte a secretaria@investigacionenlaescuela.es

La revista Investigación en la Escuela desde su origen en 1987 hasta su no 87 (2015) fue editada por Díada Editora. 
